Pacific Journal of Mathematics

INDUCED TOPOLOGIES FOR QUASIGROUPS AND LOOPS 


\title{
INDUCED TOPOLOGIES FOR QUASIGROUPS AND LOOPS
}

\author{
Kenneth BaClawski and Kenneth M. KapP
}

The concept of a [semi] topological quasigroup is defined and the notions of induced groupoids and isotopes are extended to the topological case. Necessary and sufficient conditions are found in order for a continuously induced isotope of a semitopological quasigroup to be a semitopological quasigroup.

Given an injection $i$ of a topological space $(A, \mathscr{A})$ into a set $S$ acted on by a group, $G$, a topology $\mathscr{T}_{A}$ on $S$ is introduced in a natural fashion under which $i$ is continuous. When $S=Q$ is itself a semitopological quasigroup and $G$ is generated by the left or right translations of $Q$ the continuity or openness of $i$ can be checked by comparing the topology $\mathscr{T}_{A}$ with that of $Q$. In particular this method is applied in $\S 3$ to the study of topologically invariant subloops.

1. Preliminaries; continuously induced isotopes. We recall (cf. [2]) that a quasigroup $(Q, \cdot)$ is a closed binary system i.e., a groupoid, where for any $q \in Q$ the left and right translations $\lambda_{q}, \rho_{q}$, both define bijections on $Q$. Thus given a quasigroup $(Q, \cdot)$ we can define (cf. [2], [7]) the so called conjugate operations $/, \mid$ on the set $Q$ by $c / b=a$ iff $a b=c$ iff $a \backslash c=b$ for $a, b, c \in Q$. In the conjugate quasigroups $(Q, /)$ and $(Q, \downarrow)$ we will denote the left and right translations determined by $q \in Q$ by $\lambda_{q}^{\prime}, \rho_{q}^{\prime}$, $\lambda_{q}^{\prime}$, and $\rho_{q}^{\backslash}$ respectively. (In general, $\rho_{a}^{*}$ will denote the right translation determined by $\alpha$ relative to the binary operation*.) A loop is a quasigroup with a two sided identity. A conjugate operation need not be associative; viz. if $G=(Z,+)$, where $Z$ is the set of integers then $c / b=c-b$.

We now make the following definition (cf. [4], [5]):

DeFINITION 1.1. 1. A semitopological quasigroup [loop] is a triple $(Q, \cdot, \mathscr{T})$ where $(Q, \cdot)$ is a quasigroup [loop] and $(Q, \mathscr{T})$ a Hausdorff space in which the left and right (multiplications) translations, $\lambda_{a}$, $\rho_{b}, a, b \in Q$ are homeomorphisms of $Q$ onto itself. We will write $Q=$ $(Q, \cdot ; \mathscr{S})$ is a s.t.q. [s.t.l.] in such cases.

2. A triple $(Q, \cdot ; \mathscr{T})$ is a topological quasigroup [loop] if $Q$ is a quasigroup [loop] and multiplication in $Q$ and each of its conjugates is continuous in both variables with respect to the same topology $\mathscr{T}$. We will write $Q$ is a t.q. [t.l.] in such cases.

One makes the obvious genralizations of a [semi] topological group (s.t.g.) ([5], p. 28) to [semi] topological semigroup (s.t.s.) and groupoid (s.t.gd.). We observe that a s.t.q. is homogeneous and that a s.t.g. 
is also a s.t.q.

It is easily verified that a topological quasigroup, $Q$, is a semitopological quasigroup; indeed each conjugate of $Q$ is a topological quasigroup and hence also a semitopological quasigroup. Moreover a topological group is a topological quasigroup and conversely any topological quasigroup which is algebraically a group is a topological group. (For proof, one need only consider the appropriate translations in a conjugate quasigroup, e.g., $\lambda_{a} \lambda_{a}=1_{Q}$ while $g h^{-1}=g / h$ in a group $\left.G.\right)$

We now extend the definitions of topism, etc., in [6] to involve the topological structure of given s.t.gd.'s:

Definition 1.2. 1. Let $(A, \cdot ; \mathscr{A})$ and $(B, * ; \mathscr{B})$ be two s.t.gd.'s. A triple of maps $(\gamma ; \alpha, \beta)$ from $A$ into $B$ will be called a continuous topism [isotopy] if $\gamma, \alpha$, and $\beta$ are continuous maps [and bijections] from $A$ into $B$ and $(x \cdot y)^{\gamma}=x^{\alpha} * y^{\beta}$ for all $x, y \in A$. We will say that $(\gamma ; \alpha, \beta)$ is a topological isotopy if in addition $\gamma, \alpha$, and $\beta$ are homeomorphisms.

2. Let $(A, \cdot ; \mathscr{A})$ be a s.t.gd. and $\mu, \nu$ continuous maps of $A$ into itself. The continuously induced groupoid $(A, \circ ; \mathscr{A})=(A, \bullet ; \mu, \nu ; \mathscr{A})$ of $A$ is the groupoid in which $x \circ y=x^{\mu} y^{\nu}$ for each $x, y \in A$. If $\mu$ and $\nu$ are in addition bijections [homeomorphisms] we will say that $(A, \circ ; \mathscr{A})$ is a continuously [topologically] induced (principal) isotope of $(A, \cdot ; \mathscr{A})$.

It is easy to see that a continuously induced groupoid of a [semi] topological groupoid is itself a [semi] topological groupoid. Since it is well known that any isotope of a quasigroup is a quasigroup one might readily expect that a similar result is true for continuous isotopes of semitopological quasigroups. The following example shows that this need not be the case even when one starts out with a topological group.

EXAMPLE 1.3. Let $Q=(Q,+; \mathscr{T})$ where $Q$ is the set of rational numbers and $\mathscr{T}$ the topology inherited from the usual (open interval) topology of the real line. Let $\beta=1$, the identity map, and define $\alpha$ as follows:

(a) $\alpha$ is the identity on $Q \cap(\sqrt{2}, \infty)$

(b) $\alpha$ maps $Q \cap(-\infty,-\sqrt{2})$ continuously and strictly order preserving onto $Q \cap(-\infty, 0)$

(c) $(0) \alpha=0$

(d) on $[Q \cap(-\sqrt{2}, \sqrt{2})] \backslash\{0\}=\left[\bigcup_{n=1}^{\infty} Q \cap(\sqrt{2} /(n+1), \sqrt{2} / n)\right] \cup$ [U $\left.\bigcup_{n=1}^{\infty} Q \cap(-\sqrt{2} / n,-\sqrt{2} /(n+1))\right]$ by mapping

(i) $Q \cap(\sqrt{2} /(n+1), \sqrt{2} / n)$ continuously and strictly order preserving onto $Q \cap(\sqrt{2} / 2 n, \sqrt{2} /(2 n-1))$

(ii) $Q \cap(-\sqrt{2} / n,-\sqrt{2} /(n+1))$ continuously and strictly order 
preserving onto $Q \cap \sqrt{2} /(2 n+1), \sqrt{2} / 2 n)$.

(We observe that such continuous strictly order preserving maps do exist.)

Thus $\alpha$ maps alternatively the disjoint covering of $[Q \cap$ $(-\sqrt{2}, \sqrt{2})] \backslash\{0\}$ by $\left\{L_{n}=Q \cap(-\sqrt{2} / n,-\sqrt{2} /(n+1)), R_{m}=Q \cap\right.$ $(\sqrt{2} /(m+1), \sqrt{2} / m)\}_{n, m=1}^{\infty}$ onto $Q \cap(0, \sqrt{2})$. One readily checks that $\alpha$ is a continuous bijection.

Now the continuously induced groupoid $\left(Q, \circ ; \mathscr{T}^{-}\right)=(Q,+; \alpha, \beta$; $\mathscr{T})$, which is an algebraic quasigroup, is not a semitopological quasigroup since the right translation $\rho_{0}^{\circ}$ is not an open map in $(Q, \circ ; \mathscr{T})$. We have for $a \in Q \quad a \circ O=a^{\alpha}+O^{\beta}=a^{\alpha}$ so that $[Q \cap(-\sqrt{2}, \sqrt{2})] \rho_{0}^{\circ}=$ $Q \cap[0, \sqrt{2})$ which is not open in $(Q, \mathscr{T})$.

Theorem 1.4. Let $(Q, \circ, \mathscr{T})=(Q, \cdot ; \alpha, \beta ; \mathscr{T})$ be a continuously induced isotope of a s.t.q. $(Q, \cdot ; \mathscr{T})$. If there exist $b, c \in Q$ such that $\rho_{b}^{\circ}, \rho_{c}^{\circ}$ are homeomorphisms then $(Q, \circ ; \mathscr{T})$ is a topologically induced isotope of $(Q, \cdot ; \mathscr{T})$ and $(Q, \circ ; \mathscr{T})$ is a s.t.q.

Proof. We will show that $\alpha^{-1}$ is continuous and hence a homeomorphism. Let $b \in Q$ for which $\rho_{b}^{\circ}$ is a homeomorphism. Then for $x \in Q:(x) \rho_{b}^{\circ}=x \circ b=x^{\alpha} b^{\beta}=(x) \alpha \rho_{b \beta}^{\circ}$, hence $\alpha \rho_{b \beta}^{\circ}=\rho_{b}^{\circ}$. Thus $a^{-1}=\rho_{b \beta}^{\circ}\left(\rho_{b}^{\circ}\right)^{-1}$ and the first claim is verified. Dually $\beta^{-1}=\lambda_{c^{\alpha}}^{\circ}\left(\lambda_{c}^{\circ}\right)^{-1}$ where $\lambda_{c}^{\circ}$ is the given homeomorphism.

In a similar manner one calculates that $\rho_{a}^{\circ}=\alpha \rho_{a, s}^{\circ}$ and $\lambda_{a}^{\circ}=\beta \lambda_{a^{\alpha}}^{\circ}$ for any $a \in Q$. Since an isotope of a quasigroup is a quasigroup it now follows that $(Q, \circ ; \mathscr{T})$ is s.t.q.

Corollary 1.5. Let $(Q, \cdot ; \mathscr{T})$ be a s.t.q. and $(Q, \circ ; \mathscr{T})=(Q$, $\cdot ; \alpha, \beta ; \mathscr{S})$ be a continuously induced isotope of $Q$. Then the following are equivalent:

1. $(Q, \circ ; \mathscr{T})$ is a s.t.q.

2. there exists $b, c \in Q$ such that $\lambda_{c}^{\circ}$ and $\rho_{b}^{\circ}$ are homeomorphisms.

3. $(Q, \circ ; \mathscr{T})$ is a topologically induced isotope of $Q$.

Example (1.3) shows that if $(Q, \circ)$ is not a loop then $(Q, \circ ; \mathscr{T})$ need not be s.t.q. However the following corollary is valid since the loop identity $e$ determines the homeomorphisms $\lambda_{e}^{\circ}=\rho_{e}^{\circ}$.

Corollary 1.6. If a continuously induced isotope of a s.t.q. is a loop, then it is a s.t.l.

We comment in conclusion that many of the results of [6], $\S 2$ have topological analogues. 
2. Translation topology; embeddings of semitopological quasigroups. The algebraic problem of when a binary system of one type can be embedded in a binary system of another type has in many cases been solved. Difficulties arise, however, when the algebraic systems have in addition a topological structure. Then there is the added problem of finding an embedding which is also a topological homeomorphism. Indeed, it is also often desirable to have the image open in the second space. Solutions are then dependent on the relationship between the topologies of the two structures. This problem, it will be seen, includes the more limited one of comparing with the given topology of the image space a topology induced by the given injection from the topology of the domain space.

Definition 2.1. 1. An ordered 5-tuple $(i ; A, \mathscr{A} ; S, G)$ is called a system for an induced topology of $S$ if

1. $(A, \mathscr{A})$ is a topological space,

2. $G$ is a group acting on the nonempty set $S$ and

3. $i$ is an injection of $A$ into $S$.

In such a case we will say that $(i ; A, \mathscr{A} ; S, G)$ is an s.i.t. for brevity.

2. Let $\mathscr{T}_{A}=\left\{B \subseteq S \mid(B g) i^{-1} \in \mathscr{A}\right.$ for each $\left.g \in G\right\}$. Here $(B g) i^{-1}=$ $\{a \in A \mid(a) i \in B g\} . \quad \mathscr{T}_{A}$ is called the induced action topology with respect to the s.i.t. $(i ; A, \mathscr{A} ; S, G)$.

One readily checks that $\mathscr{T}_{A}$ is a topology on $S$; it is not necessarily Hausdorff even when $A$ is Hausdorff. If $A \subseteq S$ and $i$ is the inclusion map, we see that $\mathscr{T}_{A}=\{B \subseteq S \mid B g \cap A \in \mathscr{A}$ for each $g \in G\}$.

ExAMPles 2.2. 1. Let $(A, \mathscr{A})$ be a discrete space. Then $\mathscr{T}_{A}$ for any s.i.t. is also discrete.

2. Let $(i ; A, \mathscr{A} ; S,\{e\})$ be a s.i.t. Then $\mathscr{T}_{A}=\left\{B \subseteq S \mid B i^{-1} \in \mathscr{A}\right\}$. Thus $\mathscr{T}_{A}$ contains the set of all subsets of $S \backslash(A) i$ as well as $\{(U) i \mid U \epsilon$ $\mathscr{A}\}$. Whence $\left(S, \mathscr{T}_{A}\right)$ is Hausdorff precisely when $(A, \mathscr{A})$ is Hausdorff.

3. Let $(i ; R, \mathscr{R} ; C, G)$ be a s.i.t. where $(R, \mathscr{R})$ is the real line with the usual topology, $C$ the set of complex numbers, $i$ the natural inclusion map of $R$ into $C$ and $G$ the group of all bijections on $C$. One checks that $\mathscr{T}_{R}=\{B \subseteq C \mid C \backslash B$ is finite or $B=\varnothing\}$. Clearly $\mathscr{T}_{R}$ is not a Hausdorff topology for $C$.

One sees immediately that when $(i ; A, \mathscr{A} ; S, G)$ is a s.i.t. then $i:(A, \mathscr{A}) \rightarrow\left(S, \mathscr{T}_{A}\right)$ is continuous and each $g \in G$ determines a homeomorphism of $\left(S, \mathscr{T}_{A}\right)$ onto itself. Thus if $G$ acts transitively on $S$ the space $\left(S, \mathscr{T}_{A}\right)$ is homogeneous. Moreover $\mathscr{T}_{A}$ is the strongest 
topology on $S$ under which the map $i \circ g: A \rightarrow S$ is continuous for each $g \in G$.

Following Bruck ([2], p. 54) we make the following definition:

Definition 2.3. Let $(Q, \cdot)$ be a quasigroup. We set $\mathscr{H}_{\rho}=\mathscr{L}_{\rho}(Q)$, $\mathscr{C}_{\lambda}=\mathscr{C}_{\lambda}(Q)$ and $\mathscr{C}=\mathscr{C}(Q)$, the groups, respectively, generated by the right, left, and both right and left translations of $Q$. Thus, e.g., $\mathscr{L}_{\rho}$ is generated by $\left\{\rho_{g} \mid g \in Q\right\}$.

It is clear that for a quasigroup $(Q, \cdot)$ the three groups of translations are transitive groups acting on $Q$. Thus if $(i ; A, \mathscr{A} ; Q, G)$ is a s.i.t. where $G=\mathscr{H}(Q)\left[\mathscr{L}_{\rho}(Q)\right.$ or $\left.\mathscr{C}_{\lambda}(Q)\right]$ we will speak of the [right, left] translation topology, $\mathscr{T}_{A}$, of $Q$.

THEOREM 2.4. Let $(Q, \cdot ; \mathscr{T})$ be a s.t.q. and $(i ; A, \mathscr{A} ; Q, G) a$ s.i.t. where $G=\mathscr{M}(Q)\left[\mathscr{H}_{\rho}(Q), \mathscr{M}_{\lambda}(Q)\right]$. Then

1. $i$ is continuous iff $\mathscr{T} \cong \mathscr{T}_{A}$

2. if $i$ is open then $\mathscr{T} \supseteqq \mathscr{T}_{A}$ and

3. if $i$ is an open embedding then $\mathscr{T}=\mathscr{T}_{A}$.

Proof. If $\mathscr{T} \subseteq \mathscr{T}_{A}$ then $i$ is clearly continuous. Conversely, suppose $i$ is continuous. Let $B \in \mathscr{T}$. Since for each $g \in Q, \rho_{g}, \lambda_{g}$ are homeomorphisms, $G$ is in each case a group of homeomorphisms on Q. Thus if $t \in G,(B) t=B t \in \mathscr{T}$. Whence by the continuity of $i$, $(B t) i^{-1} \in \mathscr{A}$ for each $t \in G$ and thus $B \in \mathscr{T}_{A}, \mathscr{T} \subseteq \mathscr{T}_{A}$ and the proof of 1 is complete.

Let us now suppose that $i$ is a $\mathscr{T}$-open map. Let $B \in \mathscr{T}_{A}, B \neq \varnothing$. Let $b \in B$. We will find a $\mathscr{T}$-open neighborhood of $b$ contained in $B$. Suppose $G=\mathscr{A}_{\rho}(Q)$; the proofs for the other cases are analogous. Let $a \in(A) i$. There is a unique $q \in Q$ such that $b q=a$. Now $(a) i^{-1} \in$ $(B q) i^{-1} \in \mathscr{A}$ by definition of $\mathscr{T}_{A}$. Since $i$ is open $a \in\left((B q) i^{-1}\right) i=U=$ $B q \cap(A) i \in \mathscr{T}$. Since $\rho_{q}$ is a homeomorphism in $(Q, \cdot ; \mathscr{T})$ it follows that $b=(a)\left(\rho_{q}\right)^{-1} \in(U)\left(\rho_{q}\right)^{-1}=(B q \cap(A) i)\left(\rho_{q}\right)^{-1} \subseteq B$, and $(U)\left(\rho_{q}\right)^{-1}$ is the desired $\mathscr{T}$-open neighborhood. Whence $B \in \mathscr{T}$ and $\mathscr{T}_{A} \subseteq \mathscr{T}$.

The third result is an immediate consequence of the first two and the proof is now complete.

From the results following (2.2) we now see that when $(i ; A, \mathscr{A}$; $Q, G)$ is a s.i.t. where $Q$ is a quasigroup and $G=\mathscr{C}(Q)$, if $\left(Q, \mathscr{T}_{A}\right)$ is a Hausdorff space then $\left(Q, \cdot ; \mathscr{T}_{A}\right)$ is a s.t.q.

3. Semitopological loops. We will now apply the concepts of action and translation topologies to the study of invariant subloops of a loop.

The first two parts of the following definitions can be found in Bruck ([2], p. 61). 
Definition 3.1. 1. Let $(Q, \cdot)$ be a loop with identity $e$. The subgroup of $\mathscr{C}$ defined by $\mathscr{J}=\mathscr{F}(Q)=\{t \in \mathscr{C} \mid(e) t=e\}$ is called the inner mapping group of $(Q, \cdot)$ and we note that $\mathscr{F}$ is the stabilizer of $e$ in $\mathscr{C}$.

2. Let $(Q, \cdot)$ be a loop and $(H, \cdot)$ a subloop of $Q$. We say that $H$ is a normal (invariant) subloop of $Q$ if $(H) \mathscr{F} \subseteq H$ and we write $H \unlhd Q$ in such cases.

3. Let $(H, \cdot ; \mathscr{C})$ be a topological space and a subloop of $(Q, \cdot)$. We will call $H=(H, \cdot ; \mathscr{H})$ a topologically invariant subloop of $Q$ if for each $K \in \mathscr{H}$ and $j \in \mathscr{F}, K j \in \mathscr{H}$. We write $H \unlhd_{t} Q$ in such cases and note that if $H \unlhd_{t} Q$ then $H \unlhd Q$.

THEOREM 3.2. Let $(H, \cdot ; \mathscr{H})$ be a s.t.l. and suppose $H \unlhd Q$ where $(Q, \cdot)$ is loop. The followings are then equivalent:

1. $H \unlhd_{t} Q$

2. $\mathscr{H} \subseteq \mathscr{T}_{H}$ where $\mathscr{T}_{H}$ is the action topology generated from the s.i.t. $(i ; H, \mathscr{H} ; Q, \mathscr{M}(Q))$.

3. There is a topology $\mathscr{T}$ for $Q$ under which $(Q, \cdot ; \mathscr{T})$ is a s.t.l. with $(H, \mathscr{H})$ as a subspace.

4. There is a topology $\mathscr{T}$ for $Q$ under which $(Q, \cdot ; \mathscr{T})$ is a s.t.l. with $(H, \mathscr{X})$ as an open subspace.

Proof. Assume 1. $H \unlhd_{t} Q . \quad(\mathscr{L}=\mathscr{L}(Q))$ is defined in (2.3)). Let $K \in \mathscr{H}$ and $t \in \mathscr{l l}$. We will show that $K t \cap H \in \mathscr{H}$. We assume for the sake of argument that $K t \cap H \neq \varnothing$. If $h \in K t \cap H$ then $h=$ (k) $t$ for some $k \in K$. It is easily checked that $\rho_{k} \circ t \circ\left(\rho_{h}\right)^{-1} \in \mathscr{F}$. Whence ( $H) \rho_{k} \circ t \circ\left(\rho_{h}\right)^{-1} \subseteq H$ and $\left(H \rho_{k}\right) t \subseteq H \rho_{h}$. It follows, since $H$ is a closed subloop that $(H) t \leqq H$. In particular $K t \subseteq H$ and $K t \cap H=K t$.

Now let $m=(e) t$. Then $t \circ \rho_{m}^{-1} \in \mathscr{J}$ so that $(K) t \circ \rho_{m}^{-1}=(K t) \rho_{m}^{-1} \in$ $\mathscr{H}$. But $H t \subseteq H$, a s.t.l., thus $m \in H$ and $\rho_{m}$ is a homeomorphism on $H$. It now follows that $K t \in \mathscr{H}$. Whence $K \in \mathscr{T}_{H}$ and $1 \rightarrow 2$. (We also note that if $G$ is any subgroup of $\mathscr{C}$ then $\mathscr{C} \cong \mathscr{T}_{H}^{\prime}$ where $\mathscr{T}_{H}^{\prime}$ is generated from the s.i.t. $(i ; H, \mathscr{H} ; Q, G)$.)

Assume 2 now holds, i.e., $\mathscr{\mathscr { C }} \subseteq \mathscr{T}_{H}$. Then $i:(H, \mathscr{C}) \rightarrow\left(Q, \mathscr{T}_{H}\right)$, which is continuous by Theorem (2.4), is an open embedding. Thus we need only show that $\mathscr{T}_{H}$ is a Hausdorff topology for $Q$ and 4 will follow. Thus let $a, b \in Q$ with $a \neq b$. If $H a \cap H b=\varnothing$ we are done since $H a, H b \in \mathscr{T}_{H}$. But if $H a \cap H b \neq \varnothing$ then $H a=H b$ since $H \unlhd$ $Q$. Thus $a=h b$ for some $h \in H \backslash\{e\}$. Now $(H, \mathscr{C})$ is Hausdorff so there are disjoint sets $A$ and $B$ such that $e \in B \in \mathscr{H}$ and $h \in A \in \mathscr{H}$. It follows that $b \in B b$ and $a \in A b$. But $A b \cap B b=\varnothing$ and $A b, B b \in \mathscr{T}_{H}$ since $\rho_{b}$ is a homeomorphism. Whence $\left(Q, \mathscr{T}_{H}\right)$ is Hausdorff and the 
proof of $2 \rightarrow 4$ is complete.

Clearly $4 \rightarrow 3$. We will now prove the implication $3 \rightarrow 1$.

Assume 3 holds and let $j \in \mathscr{J}(Q)$ and $K \in \mathscr{H}$. Since $H \unlhd Q$ we have $H j=H$. Now $K=H \cap U$ for some $U \in \mathscr{T}$. Thus $K j=(H \cap U) j=$ $H j \cap U j=H \cap U j$. Since $j$ is a homeomorphism of $Q, U j \in \mathscr{T}$. Hence $K j \in \mathscr{H}$ and the proof of this implication and the theorem is complete.

CoRollary 3.3. Let $(Q, \cdot ; \mathscr{T})$ be a s.t.l. The component $E$, of the identity, $e$, with the restricted product and inherited topology is a topologically invariant subloop of $Q$.

Proof. We need only show that $E \unlhd Q$. Connectivity is preserved under continuous maps so that for each $t \in \mathscr{M}(Q), E t$ is connected. Thus either $E t \cap E=\varnothing$ or $E t \leqq E$. In the latter case $E \leqq$ $E t^{-1}$ and since $t^{-1} \in \mathscr{C}(Q)$ it also follows that $E t^{-1} \subseteq E$. Whence $E=$ $E t=E t^{-1}$. Now for any $g, h \in E$ we have $g \in E \cap E \rho_{g}$ and $h \in E \cap$ $E \lambda_{h}$. Thus $E=E \rho_{g}=E \rho_{g}^{-1}=E \lambda_{h}=E \lambda_{h}^{-1}$ and it follows that $E$ is closed. In particular for any $j \in \mathscr{J}(Q), e=(e) j, E \cap E \cap E_{j} \neq \varnothing$ so that $E j=E$ and hence $E \leqq Q$. The result now follows from Theorem (3.2).

Definition 3.4. Let $(H, \cdot ; \mathscr{H})$ be a s.t.l. and a subloop of a loop $(Q, \cdot)$. Then $R_{H}=\left\{B t \mid B \in \mathscr{H}, t \in \mathscr{L}_{\rho}(Q)\right\}$ and $L_{H}=\{C s \mid C \in \mathscr{H}$, $\left.s \in \mathscr{L}_{2}(Q)\right\}$ are called, respectively, the right and lest coset coverings of $Q$ with respect to $(H, \cdot ; \mathscr{H})$.

Theorem 3.5. Let $H \unlhd_{t} Q$ where $(H, \cdot ; \mathscr{K})$ is a s.t.l. then $R_{H}=$ $L_{H}$ is a subbase for a topology $\mathscr{R}_{H}=\mathscr{L}_{H}$ under which $(Q, \cdot)$ is a s.t.l. Moreover if $i$ is the natural inclusion map of $H$ into $Q$ and $G=\mathscr{L l}(Q)\left[\mathscr{C l}_{\rho}(Q), \mathscr{C l}_{\lambda}(Q)\right]$ then $\mathscr{T}_{H}=\mathscr{R}_{H}=\mathscr{L}_{H}$, where $\mathscr{T}_{H}$ is generated from the s.i.t. $(i ; H, \mathscr{X} ; Q, G)$, and $i$ embeds $(H, \mathscr{Y})$ as an open subspace of $\left(Q, \mathscr{T}_{H}\right)$.

Proof. Let $B \in \mathscr{H}$ and $t \in \mathscr{L}_{\rho}(Q)$. Set $m=(e) t$. Then $t \circ \lambda_{m}^{-1} \in$ $\mathscr{F}$ so that $(B) t \circ \lambda_{m}^{-1} \in \mathscr{\mathscr { C }}$ since $H \unlhd_{t} Q$. Thus $B t=\left((B) t \circ \lambda_{m}^{-1}\right) \lambda_{m} \in L_{H}$ and $R_{H} \subseteq L_{H}$. Similarly $L_{H} \subseteq R_{H}$ and equality now follows. Clearly $\cup R_{H}=Q$ so that $R_{H}$ is a subbase for a topology on $Q$.

We will show that $\left(Q, \mathscr{R}_{H}\right)$ is Hausdorff. Let $q_{1} \neq q_{2} \in Q$. Since $H \unlhd Q$ (cf. [2], pp. 60, 61) either $H q_{1}=H q_{2}$ or $H q_{1} \cap H q_{2}=\varnothing$. In the latter case, since $H q_{1}, H q_{2} \in R_{H}$ and $e \in H$ we have two disjoint open neighborhoods in $\mathscr{R}_{H}$ of $q_{1}$ and $q_{2}$. Suppose now that $H q_{1}=H q_{2}$. Then $q_{1}=h q_{2}$ for some $h \in H \backslash\{e\}$, where $e$ is the loop identity. Since $(H, \mathscr{C})$ is Hausdorff there are open disjoint neighborhoods, $A, B \in$ 
$\mathscr{H}$ of $e$ and $h$ respectively. Then $A q_{2} \cap B q_{2}=(A \cap B) q_{2}=\varnothing, q_{2} \in A q_{2}$ and $q_{1} \in B q_{2}$. But $A q_{2}, B q_{2} \in R_{H}$ and it follows that $\left(Q, \mathscr{R}_{H}\right)$ is Hausdorff.

Now let $t \in \mathscr{M}_{\rho}(Q)$. Then if $\bigcap^{n} B_{i} t_{i}$ is a basic open set of $\mathscr{R}_{H}$ we have $\left(\cap B_{i} t_{i}\right) t=\cap\left(B_{i} t_{i}\right) t=\cap B_{i}\left(t_{i} t\right)$ another basic open set of $\mathscr{R}_{H}$. Thus $t$ is an open map. Since $t^{-1} \in \mathscr{M}_{\rho}$ it is also true that $t$ is continuous. Whence $t$ is a homeomorphism. Since all right translations determined by $q \in Q$ are in $\mathscr{A}_{\rho}$ it follows that each such $\rho_{q}$ is a homeomorphism. In a similar fashion since $R_{H}=L_{H}$, each $\lambda_{q}$ is a homeomorphism. Thus $\left(Q, \cdot ; \mathscr{R}_{H}\right)=\left(Q, \cdot ; \mathscr{L}_{H}\right)$ is a s.t.q.

We have already seen in the proof of Theorem (3.2) that $\mathscr{H} \subseteq$ $\mathscr{T}_{H}$ where $\mathscr{T}_{H}$ is generated from the s.i.t. $(i ; H, \mathscr{H} ; Q, G)$ and $G=$ $\mathscr{C}\left[\mathscr{M}_{\rho}, \mathscr{C}_{\lambda}\right]$. Let $B t \in R_{H}$ where $B \in \mathscr{H}, t \in \mathscr{M}_{\rho}$. Since $t$ is a homeomorphism and $\mathscr{H} \subseteq \mathscr{T}_{H}$ it follows that $B t \in \mathscr{T}_{H}$ and thus $\mathscr{R}_{H} \subseteq \mathscr{T}_{H}$. Conversely since the inclusion map $i: H \rightarrow Q$ is open for the s.t.l. $\left(Q, \cdot ; \mathscr{R}_{H}\right)$ by Theorem $(2.4 .2)$ we have $\mathscr{R}_{H} \supseteqq \mathscr{T}_{H}$ and equality follows. The other assertions are now clear and the proof is complete.

The following corollary is now an immediate consequence of the above and Theorem (2.4).

Corollary 3.6. Let $(H, \cdot ; \mathscr{H})$ and $(Q, \cdot ; \mathscr{T})$ be two s.t.l.'s. with $H \unlhd_{t} Q$. If $i$ is the natural inclusion map of $H$ into $Q$ then

1. $i$ is continuous iff $\mathscr{T} \cong \mathscr{R}_{H}$

2. $i$ is open iff $\mathscr{T} \supseteqq \mathscr{R}_{H}$

3. $i$ is an opening embedding iff $\mathscr{T}=\mathscr{R}_{H}$.

We conclude with an example which illustrates both the existence of a normal subloop of a s.t.l. which is not topologically invariant. The example will show that necessity of the topological invariance in the hypothesis of several results viz. Theorem (3.2), and Theorem (3.5).

ExAmple 3.7. Let $C$ be the set of complex numbers. For $m \epsilon$ $\left(Z_{2},+\right)$ we define an action of $Z_{2}$ on $C$ by $z^{m}=\left\{\begin{array}{l}z \\ \bar{z} \text { if } m=0 \\ \text { if } m=1\end{array}\right.$ where $z \epsilon$ $C$ and $\bar{z}$ denotes the conjugate $\dot{x}-y i=\overline{x+y i}=\bar{z}(x, y \in R$, the reals). It is easy to check that this is an action and that $\left(z_{1}+z_{2}\right)^{\frac{m}{}}=z_{1}^{\frac{m}{1}}+z_{2}^{\frac{m}{2}}$. We will consider $R$, the set of real numbers, with two different topologies: $\mathscr{R}$, the usual topology of the real line, and $\mathscr{S}$, the Sorgenfrey topology (generated by half open intervals). We can now define the following two topologies on $C$ where we make the usual identification $x+y i \rightarrow(x, y)$. The usual topology generated from $\mathscr{R} \times$ $\mathscr{R}$ will be denoted by $\mathscr{C}$ while we will denote by $\mathscr{C}^{\prime}$ the cartesian product topology generated from $\mathscr{R} \times \mathscr{S}$. It is readily checked that 
$(C,+; \mathscr{C})$ and $\left(C,+; \mathscr{C}^{\prime}\right)$ are both semitopological groups.

Let $P=C \times Z_{2}$. We construct a group on $P$ by defining a binary operation as follows:

$$
(w, m)+(z, n)=\left(w+z^{\underline{m}}, m+n\right) \quad \text { for } \quad(w, m),(z, n) \in P .
$$

A straightforward check shows that $(P,+)$ is a group which contains the subgroup $C \times\{0\}$. One readily checks, for example, that $(z, m)+\left(-z^{m}, m\right)=(0,0)$ for any $(z, m) \in P$ and that $(0,0)$ is the identity of $P$. We identify now $C$ and $C \times\{0\}$ since they are isomorphic under the obvious natural map. It is easily seen that $[P: C]=$ 2 and thus $C \unlhd P$. Furthermore $(z, 0)+(0,1)=(z, 1) \neq(\bar{z}, 1)=(0,1)+$ $(z, 0)$ so that $P$ is not abelian. However $(\bar{z}, 0)=(0,1)+(z, 0)+0,1)=$ $(z, 0) i_{(0,1)}$. Thus, the inner automorphism determined by $(0,1)$ when restricted to $C$ is just conjugation on $C$. Now if we consider the semitopological loop $\left(C,+; \mathscr{C}^{\prime}\right)$ as a subloop of $(P,+)$ we see that $\left(C,+; \mathscr{C}^{\prime}\right)$ is a normal but not a topologically invariant subloop, since conjugation in $\left(C, \mathscr{C}^{\prime}\right)$ is not a continuous map.

Let $\left(i ; C, \mathscr{C}^{\prime} ; P, \mathscr{C}\right)$ be a s.i.t. We shall show that the topology inherited by $(C) i$ from $\mathscr{T}_{C}$ is exactly $\mathscr{C}$. It will then follow that $i$ is not open or even an embedding so that the converses of (2.4.2) and (2.4.3) are false when we take $\left(P,+; \mathscr{T}_{C}\right)$, i.e., $\mathscr{T}=\mathscr{T}_{C}$, in the hypothesis. Now let $K \subseteq P$. Since $P=C \cup[C+(0,1)]$ we can decompose $K$ into two subsets $K=K_{1} \cup\left[K_{2}+(0,1)\right]$ where $K_{i} \subseteq C$. Since $(P,+)$ is a group $\mathscr{C}(P)=\left\{\lambda_{q_{1}} \rho_{q_{2}} \mid q_{i} \in P\right\}$ and $K \in \mathscr{T}_{c}$ iff $[(w, m)+$ $K+(z, n)] \cap C \in \mathscr{C}^{\prime} . \quad$ But $(w, m)+K+(z, n)=\left(w+K_{1}^{m}+z^{m}, m+n\right) \cup$ $\left(w+K_{\frac{m}{2}}+z^{1+m}, 1+m+n\right)$. Thus if we intersect with $C=C \times\{0\}$ we get either $w+z^{m}+K_{\frac{m}{1}}$ when $m=n$ i.e., if $m+n=0$ or $w+z^{1+m}+K_{\frac{m}{2}}^{m}$ when $m \neq n$ i.e., if $m+n=1$. Hence $K \in \mathscr{T}_{C}$ iff $K_{1}, \bar{K}_{1}, K_{2}, \bar{K}_{2} \in \mathscr{C}^{\prime}$ (note: $\left(C,+; \mathscr{C}^{\prime}\right)$ is a s.t.g.!) It then follows that $K_{1}$ and $K_{2} \in \mathscr{C}$. Whence a subset $K \subseteq P$ is open iff $K=K_{1} \cup\left[K_{2}+(0,1)\right]$ where $K_{i} \epsilon$ $\mathscr{C}$. Clearly $\left(P, \mathscr{T}_{c}\right)$ is Hausdorff and it follows that $\left(P,+; \mathscr{T}_{c}\right)$ is a s.t.g.

The inclusion map $i:\left(C,+; \mathscr{C}^{\prime}\right) \rightarrow\left(P,+; \mathscr{T}_{C}\right)$ is continuous but not open since the inherited topology of $(C) i$ is $\mathscr{C}$ and $\mathscr{C} \varsubsetneqq \mathscr{C}$. Indeed, $i$ is not even an embedding (cf. (2.4)) as we were to show. Theorem (3.2) is thus false if topologically is omitted from the hypothesis since here $\mathscr{C}^{\prime} \nsubseteq \mathscr{T}_{C}$.

We now remark that Theorem (3.5) does not hold in this example. If $K$ is the upper half plane including the real axis then $K \in \mathscr{C}^{\prime}$. However, if $K+(0,1)=(w, m)+L$ for some $L \in \mathscr{C}^{\prime}$ then $\left(-w^{m}, m\right)+$ $K+(0,1) \in \mathscr{C}^{\prime}$. Hence $m+1=0$ i.e., $m=1$ and we have, since $K=K \times\{0\},(-\bar{w}, 1)+K+(0,1)=-\bar{w}+\bar{K}$ which is not in $\mathscr{C}^{\prime}$. Theorem (3.5) fails for in this example neither $\mathscr{R}_{C}$ nor $\mathscr{L}_{C}$ are topologies 
under which $(P,+)$ is a s.t.g.: the inner automorphism $i_{(0,1)}$ is not continuous.

\section{REFERENCES}

1. J. Aczel, Quasigroups, Nets, and Nomograms, Advances in Mathematics, 1 (1965), Academic Press, 383-450.

2. R. H. Bruck, A Survey of Binary Systems, Ergebnisse der Math. Neue Folge, Vol. 20 Springer, 1958.

3. K. H. Hofmann, Topologische loops, Math. Z., 70 (1958), 13-37.

4. K. H. Hofmann and P. S. Mostert, Elements of Compact Semigroups, Merrill Books, Inc., Columbus, Ohio, 1966.

5. T. Husain, Introduction to Topological Groups, W. B. Saunders Co., Philadelphia, Pa., 1966.

6. K. Kapp and K. Baclawski, Topisms and induced non-associative systems, Pacific J. Math., 36 (1971), 45-54.

7. S. K. Stein, On the foundations of quasigroups, Trans. Amer. Math. Soc., 85 (1957), 228-256.

Received August 30, 1971 and in revised form September 14, 1972.

HARVARD UNIVERSITY

AND

UNIVERSITY OF WISCONSIN-MILWAUKEE 


\section{PACIFIC JOURNAL OF MATHEMATICS}

\section{EDITORS}

\author{
H. SAMELson \\ Stanford University \\ Stanford, California 94305 \\ C. R. HоввY \\ University of Washington \\ Seattle, Washington 98105
}

J. DugundJI

Department of Mathematics

University of Southern California

Los Angeles, California 90007

RICHARD ARENS

University of California

Los Angeles, California 90024

\section{ASSOCIATE EDITORS}
E. F. BeCKENBACH
B. H. NEUMANN
F. WOLF
K. YoshidA

\section{SUPPORTING INSTITUTIONS}

UNIVERSITY OF BRITISH COLUMBIA

CALIFORNIA INSTITUTE OF TECHNOLOGY

UNIVERSITY OF CALIFORNIA

MONTANA STATE UNIVERSITY

UNIVERSITY OF NEVADA

NEW MEXICO STATE UNIVERSITY

OREGON STATE UNIVERSITY

UNIVERSITY OF OREGON

OSAKA UNIVERSITY

\author{
UNIVERSITY OF SOUTHERN CALIFORNIA \\ STANFORD UNIVERSITY \\ UNIVERSITY OF TOKYO \\ UNIVERSITY OF UTAH \\ WASHINGTON STATE UNIVERSITY \\ UNIVERSITY OF WASHINGTON

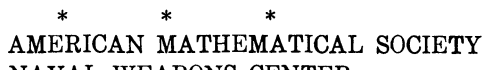 \\ NAVAL WEAPONS CENTER
}

The Supporting Institutions listed above contribute to the cost of publication of this Journal, but they are not owners or publishers and have no responsibility for its content or policies.

Mathematical papers intended for publication in the Pacific Journal of Mathematics should be in typed form or offset-reproduced, (not dittoed), double spaced with large margins. Underline Greek letters in red, German in green, and script in blue. The first paragraph or two must be capable of being used separately as a synopsis of the entire paper. The editorial "we" must not be used in the synopsis, and items of the bibliography should not be cited there unless absolutely necessary, in which case they must be identified by author and Journal, rather than by item number. Manuscripts, in duplicate if possible, may be sent to any one of the four editors. Please classify according to the scheme of Math. Rev. Index to Vol. 39. All other communications to the editors should be addressed to the managing editor, Richard Arens, University of California, Los Angeles, California, 90024.

50 reprints are provided free for each article; additional copies may be obtained at cost in multiples of 50 .

The Pacific Journal of Mathematics is issued monthly as of January 1966. Regular subscription rate: $\$ 48.00$ a year (6 Vols., 12 issues). Special rate: $\$ 24.00$ a year to individual members of supporting institutions.

Subscriptions, orders for back numbers, and changes of address should be sent to Pacific Journal of Mathematics, 103 Highland Boulevard, Berkeley, California, 94708.

PUBLISHED BY PACIFIC JOURNAL OF MATHEMATICS, A NON-PROFIT CORPORATION

Printed at Kokusai Bunken Insatsusha (International Academic Printing Co., Ltd.), 270, 3-chome Totsuka-cho, Shinjuku-ku, Tokyo 160, Japan. 


\section{Pacific Journal of Mathematics}

\section{Vol. 45, No. $2 \quad$ October, 1973}

Kenneth Paul Baclawski and Kenneth Kapp, Induced topologies for quasigroups and loops ............................................. 393

D. G. Bourgin, Fixed point and $\min -\max$ theorems $\ldots \ldots \ldots \ldots \ldots \ldots \ldots$

J. L. Brenner, Zolotarev's theorem on the Legendre symbol ............... 413

Jospeh Atkins Childress, Jr., Restricting isotopies of spheres .............. 415

John Edward Coury, Some results on lacunary Walsh series ................ 419

James B. Derr and N. P. Mukherjee, Generalized Sylow tower groups. II . . . . . . 427

Paul Frazier Duvall, Jr., Peter Fletcher and Robert Allen McCoy, Isotopy Galois

spaces .......................................... 435

Mary Rodriguez Embry, Strictly cyclic operator algebras on a Banach space ... 443

Abi (Abiadbollah) Fattahi, On generalizations of Sylow tower groups ......... 453

Burton I. Fein and Murray M. Schacher, Maximal subfields of tensor products . . 479

Ervin Fried and J. Sichler, Homomorphisms of commutative rings with unit

element .......................................... 485

Kenneth R. Goodearl, Essential products of nonsingular rings ............. 493

George Grätzer, Bjarni Jónsson and H. Lakser, The amalgamation property in

equational classes of modular lattices ...........................

507

$\mathrm{H}$. Groemer, On some mean values associated with a randomly selected simplex

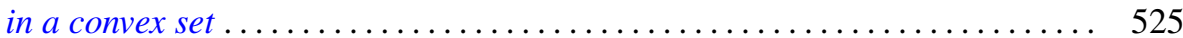

Marcel Herzog, Central 2-Sylow intersections .................... 535

Joel Saul Hillel, On the number of type-k translation-invariant groups ........ 539

Ronald Brian Kirk, A note on the Mackey topology for $\left(C^{b}(X)^{*}, C^{b}(X)\right) \ldots \ldots .543$

J. W. Lea, The peripherality of irreducible elements of lattice.............. 555

John Stewart Locker, Self-adjointness for multi-point differential operators ..... 561

Robert Patrick Martineau, Splitting of group representations ............... 571

Robert Massagli, On a new radical in a topological ring ................. 577

James Murdoch McPherson, Wild arcs in three-space. I. Families of Fox-Artin

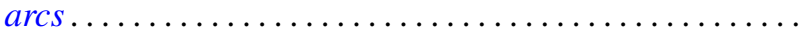

585

James Murdoch McPherson, Wild arcs in three-space. III. An invariant of

oriented local type for exceptional arcs . . . . . . . . . . . . ............ 599

Fred Richman, The constructive theory of countable abelian p-groups ........ 621

Edward Barry Saff and J. L. Walsh, On the convergence of rational functions

which interpolate in the roots of unity ..................

Harold Eugene Schlais, Non-aposyndesis and non-hereditary

decomposability..................................... 643

Mark Lawrence Teply, A class of divisible modules................... 653

Edward Joseph Tully, Jr., H-commutative semigroups in which each

homomorphism is uniquely determined by its kernel ................. 669

Garth William Warner, Jr., Zeta functions on the real general linear group ...... 681

Keith Yale, Cocyles with range $\{ \pm 1\} \ldots \ldots \ldots \ldots \ldots \ldots \ldots \ldots \ldots \ldots \ldots \ldots \ldots . \ldots \ldots$

Chi-Lin Yen, On the rest points of a nonlinear nonexpansive semigroup ........ 699 